\title{
Iris Feature Extraction and Matching by using Wavelet Decomposition and Hamming Distance
}

\author{
A. T. Gaikwad \\ Institute of Management \\ Studies and Information Technology, \\ Aurangabad, (M.S), India
}

\author{
Mouad M. H. Ali \\ Information system \&Technology Center \\ Hodeidah University, Hodeidah, Yemen
}

\begin{abstract}
The security system nowadays using biometrics traits is a confident and reliable of some biometrics system. The reason of that is uniqueness and permanents of those traits. In this paper the Iris recognition system is covering step by steps started namely Acquisition stage, preprocessing which includes the segmentation, pupil boundary detect and Normalization the next stage is feature extraction which used the wavelet decomposition methods, the finally the matching stage is perform with help of hamming distance measure, then the results are show in different dataset size of training and testing sets finally the evaluation of the system conducted based on FAR , FRR and EER of the system.
\end{abstract}

\section{General Terms}

Pattern Recognition, Computer Vision, Image processing

\section{Keywords}

Iris Recognition, preprocessing, Feature Extraction, Matching, Wavelet Decomposition, Hamming Distance.

\section{INTRODUCTION}

Biometrics Recognition refers to use one of the biometrics characteristic (biological and behavioral). The iris one of the internal parts of body can be seen from outside of the body and the iris are protected by cornea, aqueous humour and eyelid $[1,2]$. The iris is muscle which controls the amount of light to enter in the pupil. Moreover the iris cannot use after death because it's closely connected to brain and its one of the first parts of the body to decay after death [2]. The iris is rich in features to extract such as feature collage nous fibers, contraction furrows, coronas, crypts, color, serpentine vasculature and striations. While the diameter size of iris is around $12 \mathrm{~mm}$ only [3]. In 1987 two American ophthalmologists (Flow and Safir) get a patent by conjecture in iris of the human can use as identifier but they do not have an actual algorithm. Thus, in 1990 John Daugman created the first actual algorithm for iris recognition. And after a few years Daugman published the results in 1993 [4]. The data which extracted from the iris is in binary format and its store in 256 bytes. In fig 1 sample iris image with determine the parts of iris image. Iris trait relies on unique and permanence of the human to identify or verify an individual which remains stable throughout life [10]. The iris model has make attention to researchers over last 15 years [10][11][12] and it is best trait and reliable for person identification and verification system[13][16]. Iris recognition is best reliable biometric technology for verification performance and identification. Iris recognition a system are mostly used in real time application to recognize the person/ authenticates and recently used in ATM in banking, Airport in immigration. There are different ways to perform iris recognition, which include John
Daugman's renowned IrisCode [14], another used Haar decomposition and Gabor wavelet [15].

\section{Advantages}

1. Iris recognition has the smallest outlier (those who cannot use/enrol) group of all biometric technologies.

2. Template longevity is a key advantage of this technique as barring trauma, a single enrolment can last a lifetime.

3. The iris has a fine texture that is determined randomly during embryonic gestation. Even genetically identical individuals (twins) have completely independent iris textures, whereas DNA (genetic "fingerprinting") is not unique for the about $0.2 \%$ of the human population who have a genetically identical twin.

4. John Daugman's Iris Code, which is the originally commercially deployed iris recognition algorithm, has an unprecedented false match rate (FMR).

\section{Disadvantages}

1. There are few legacy databases.

2. The small size of the iris makes sampling of the iris pattern require a great deal of user cooperation or complex, expensive input devices.

3. The performance of iris authentication may be impaired by glasses, sunglasses and contact lenses.

4. The iris biometric is not left as evidence on crime scene so it is not useful for forensic applications.

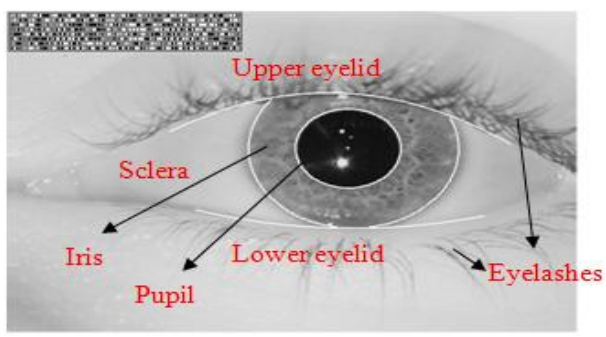

Fig 1: Iris image

The paper organized in five sections, the remaining sections, section 2 given the related work of iris recognition, in section 3 given the iris recognition process and methodology, the experimental results are given in section 4 finally, the conclusion and future work given in section 5 .

\section{RELATED WORK}

In 1987 two American ophthalmologists (Flow and Safir) get a patent by conjecture in iris of the human can use as 
identifier but they do not have an actual algorithm. Thus, in 1990 John Daugman created the first actual algorithm for iris recognition. And after a few years Daugman published the results in 1993 [4].

The data which extracted from the iris is in binary format and its store in 256 bytes. Also he is proposed the first algorithm to isolate the iris by used integro-differential operator to locate the iris region, pupil and the arcs of eyelids for segment the circle of iris region. But it has heavy computation, then it will require more time also remapped each point in the iris region into polar coordinate also he is use first algorithm to extract the iris features by using 2D Gabor wavelets at several resolution levels, and then used the Hamming distance to compare between the iris codes.

L. Masek [5] utilized a circle Hough Transform to locate the iris and pupil regions and applied the linear Hough Transform to locate the eyelids. Then the threshold is used to isolate the eyelashes and reflections. And he used the pupil center as reference point and applied remap formula to rescale the points depend on the angle around the circle.

Verma et al. [6] used Duagman's algorithm in segmentation to find the pupil and iris. By modified integro-differential operator (IDO) of Duagman algorithm to ignore the circle which it has pixel value is higher than threshold, thus it will isolated the reflections and eyelashes for a better segmentation. It applied the sobel edge detection to search the region of eyelids and the size of searching regions is equal to the diameter of pupil. Then detected the eyelids by used the linear Hough Transform. Another researcher his name Emine et al [7] utilized tow methods for segmentation; firstly, Hough transform to detected the outer boundaries of iris, and secondly integro-differential operators (IDO) to detected the inner boundaries of iris or the boundaries of pupil.

A. Oluwakemi et al. [8] proposed a FWT (fast wavelet transform) to extracted the iris features based on the iris texture. And encoded the features based on the value of each pixel; if pixel value greater than 0.5 then 11 bits is extracted, if pixel value less than 0.5 and greater than 0 then 10 is extracted, if pixel value less than 0 and greater than -0.5 then 01 is extracted, and finally if pixel value is less than -0.5 then 00 is extracted. The Hamming distance used to compare between the iris codes.

Y. Hu et al. [9] applied the Local Binary Pattern to the iris normalized image, and then used the chunk method to reduce the size of LBP image by divides it into equal size of blocks and stored the relations between local information in each block. The hamming distance used for matching and the recognition rate is $99.27 \%$.

\section{IRIS RECOGNITION PROCESS AND METHODOLOGY}

In the general the main steps of iris recognition system are show in fig .2 which including training and testing image acquisition, image preprocessing, feature extraction and finally matching

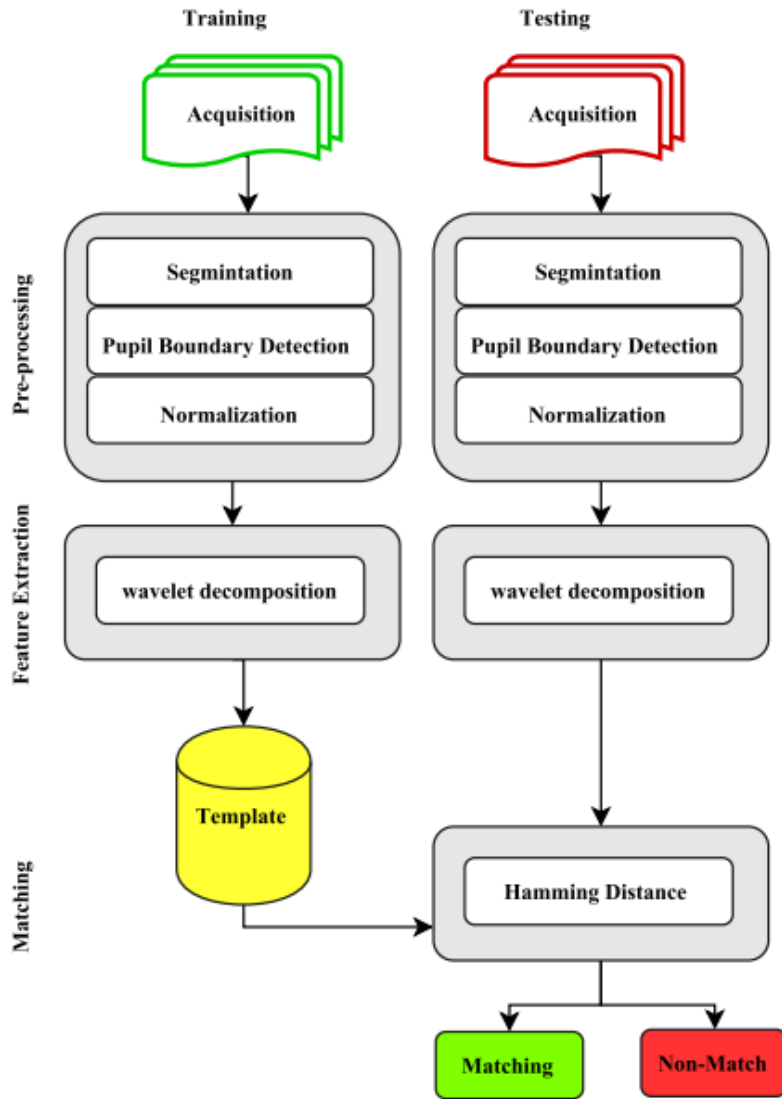

Fig 2: Iris recognition process and methodology

\subsection{Acquisition Stage}

The Acquisition stage is the process to obtain image. These images should clearly show the entire eye especially iris and pupil part. The iris image can be captured using a standard camera using both visible and infrared light and may be either a manual or automated procedure. In this work the images are collected from standard database which is available online.

\subsection{Pre-processing and Segmentation Stage}

After this step the iris images are clarity and it is necessary to obtain image points or image pixels that are on the desired curve in the image space. The segmentation in iris recognition is to isolate the actual iris region from the different parts of eye. While the iris bounded by two circlers, one for the sclera boundary and another for the pupil boundary. There are two steps to do preprocessing segmentation which are called Iris Localization and Iris Boundary Detection. The iris localization is a process to determine an iris part of the image by localizing the position of the image derived from outer boundary and inner boundary, and finally convert the iris part into a suitable representation.

\subsection{Pupil Boundary Detection Stage}

In this step are applied to determining the gray level value for each pixel in the image for the corresponding RGB components. Then, applied the binarization process by adaptively selecting the threshold, then, scans all image line by line, to find the circular pupil region and determine the outer boundary, diameter and center of the pupil.

\subsection{Normalization Stage}

It is the process to prepare the segmented region of iris for the feature extraction process. And the reasons to do iris normalization because Iris images are effect by the distance 
and the angular position from sensor/camera. And the second reason is the iris image is stretchable muscle, which it's resizing based on the difference levels of illumination. And the last reason is tilting the head.

\subsection{Feature Extraction Stage}

In these steps the output of pre-processing steps is send to the feature extraction. The feature are extract from iris image, Iris provides abundant texture information. A feature vector is formed which consists of the ordered sequence of features extracted from the various representation of the iris images. Feature extraction is presented in order to reduce the input iris data and transfers it to feature vector. In this paper the 1-D signal of the iris image is extracted by using wavelet decomposition.

\subsection{Matching Stage}

The matching stage is a process to calculate the degree of similarity between the input test image (Acquisition) and training image from the database (Enrollment).

The feature vectors are classified through different threshold techniques like hamming distance, weight vector and winner selection, dissimilarity function etc. in this work hamming distance approach is used to compare the input and the template by using Hamming Distance formula which given by Equation (1)

$\mathrm{HD}=\frac{1}{N} \sum_{J=1}^{N} C_{A}(j) \oplus C_{B}(i)$

Where $\mathrm{CA}$ and $\mathrm{CB}$ are the coefficients of two iris images, $\mathrm{N}$ is the size of the feature vector, Ex-OR is the Boolean operator that gives a binary 1 if the bits at the position $\mathrm{j}$ in $\mathrm{CA}, \mathrm{CB}$ are different and 0 if they are similar.

- $\quad$ IF HD <= Threshold then Match Pair.

- $\quad$ IF HD > Threshold then non-Match pair.

By this role the Genuine and imposter are calculated, then with help of Genuine and imposter the False Accept Rate and False Reject Rate are calculated with all this parameter the Accuracy of the system compute and find the result of the system. The steps to do the matching, the first iris code is divided into 2 subsets and the matching is done separately for each subset using Hamming Distance, and the matching is done treating the two subsets as a single entity.

\section{EXPERIMENTS AND RESULTS}

To test the performance of the system, the iris images are collected from standards database which is available online. The samples of iris image are show in fig 3 .

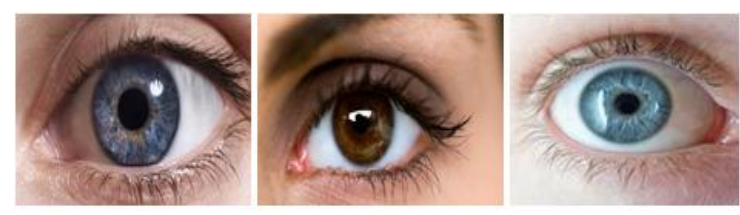

Fig 3: samples of iris image

And the pre-processing steps are applied on the iris images. The steps are called segmentation, Iris Localization and Iris Boundary Detection to isolate the actual iris region from the different parts of eye. While the iris bounded by two circlers, one for the sclera boundary and another for the pupil boundary the fig. 4 show the pre-processing steps of one sample.
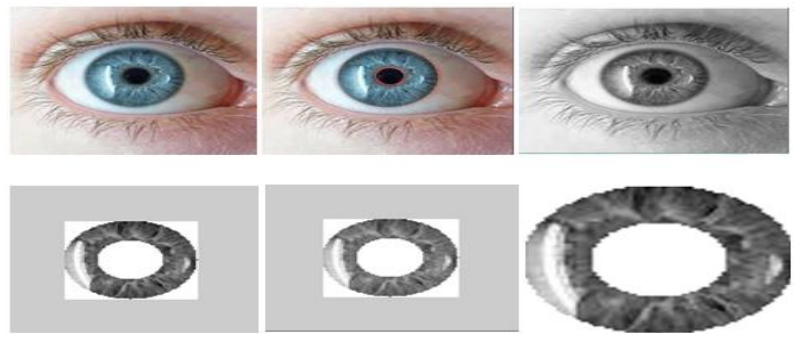

Fig 4: pre-processing steps

The feature extraction is applied on the output of preprocessing. The feature extract is done by extract the 1-D signal of the iris image fig 5 and apply signal level discrete 1D wavelet transform on iris signal vector with help of wavelet name. The Multilevel 1-D wavelet decomposition applied on levels $1,2,3$, and 4 ,then the 1-D approximation coefficients vector is compute for each level and plotted in fig 6 .

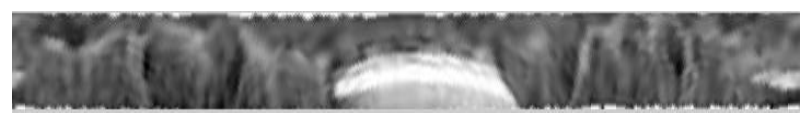

Fig 5: extract the 1-D signal of the iris image
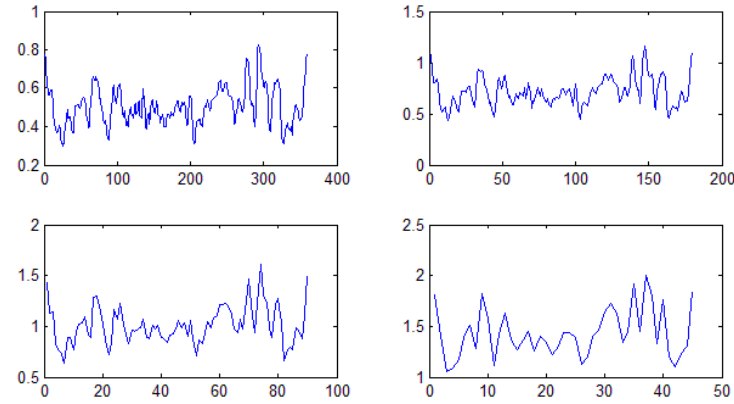

Fig 6: Wavelet Decomposition

The evaluation of the system based on False Accept Rate (FAR) and False Reject Rate (FRR) and Error Reject Rate (EER) as show in equation 2, 3 and 4 respectively

$$
\begin{aligned}
& F A R=\frac{\text { imposter score }}{\text { All imposter score }} \times 100 \\
& F R R=\frac{\text { Genuine score failing below thershold }}{\text { All Genuine score }} \times 100 \\
& \text { Accuracy }=100-\frac{F A R+F R R}{2}
\end{aligned}
$$

The Accuracy of the system is calculated for all dataset with different training set and testing set. The table 1 shows the summaries of the result getting from different training and testing set with False Accept Rate, False Reject Rate and finally the Error Reject Rate of the system with Accuracy.

Table 1.The performance of the system

\begin{tabular}{|c|c|c|c|c|c|}
\hline \multirow{2}{*}{$\begin{array}{c}\text { S } \\
\text { No. }\end{array}$} & \multicolumn{2}{|c|}{ Dataset size \% } & \multirow{2}{*}{$\begin{array}{c}\text { FAR } \\
\text { set }\end{array}$} & $\begin{array}{c}\text { Test } \\
\text { set }\end{array}$ & FRR \\
(\%) & $\begin{array}{c}\text { Accuracy } \\
\text { (\%) }\end{array}$ & \\
\hline 1 & $70 \%$ & $30 \%$ & 0.20 & 0.11 & $89.03 \%$ \\
\hline 2 & $80 \%$ & $20 \%$ & 0.41 & 0.13 & $82.55 \%$ \\
\hline 3 & $50 \%$ & $50 \%$ & 0.82 & 0.23 & $80.03 \%$ \\
\hline
\end{tabular}

From the table 1 the result of the system when the dataset divided into $70 \%$ as training and $30 \%$ as a testing the false 
accept rate and false reject rate are decrease compare with the another result of dataset divided.

The table 2 shows the execution time taken for each steps individually in millisecond (ms), start with preprocessing and feature extraction and matching finally the total of execution time are calculated.

Table 2.The Execution time of the system

\begin{tabular}{|c|c|c|}
\hline S .No. & Step & Time (ms) \\
\hline 1 & Iris Localization & 38.5 \\
\hline 2 & Normalization & 15.2 \\
\hline 3 & Generated Wavelet features & 38.6 \\
\hline 4 & Matching & 1.5 \\
\hline \multicolumn{2}{|c|}{ Total time of the system } & $\mathbf{9 3 . 8}$ \\
\hline
\end{tabular}

The performance of the system done by each iris image in dataset is match against the others to compute the genuine and impostor of the system. The matching distance distribution is shown in figure 7 .

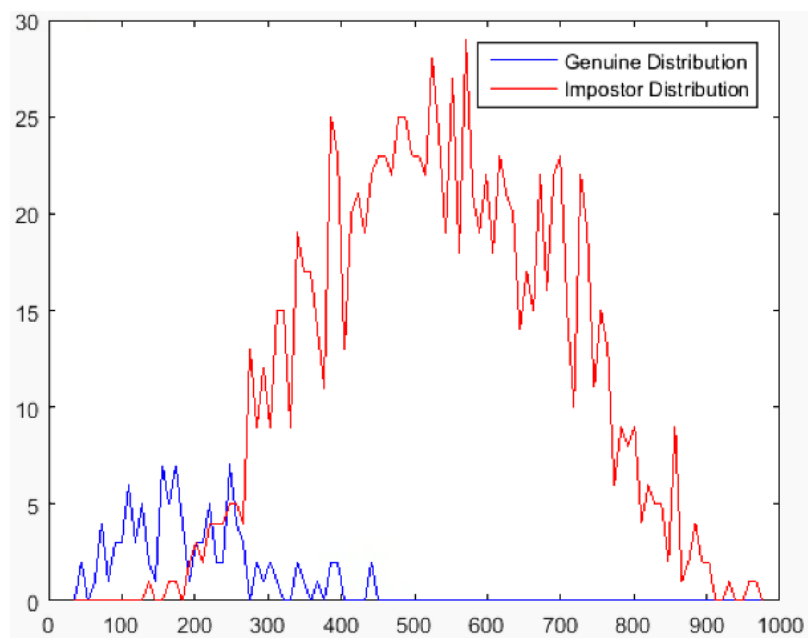

Fig 7: Matching distance distribution

The accuracy of the system is calculated with help of genuine and impostor. The FAR and FRR of the system are shown in fig 8 .

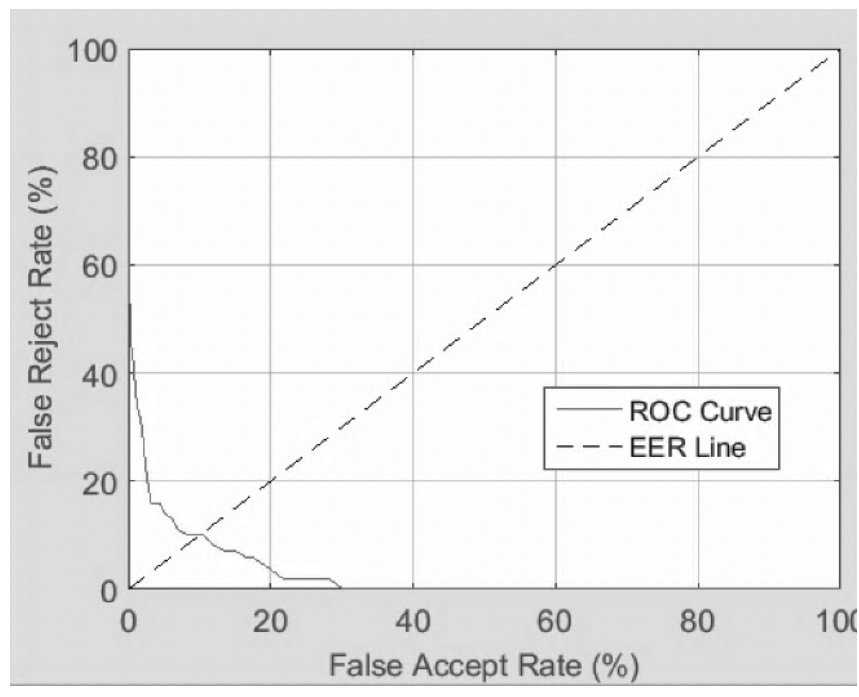

Fig 8: ROC of iris recognition system

\section{CONCLUSION AND FUTURE WORK}

In this paper the iris recognition process are conducted step by step , where the process is divided into two stages training stage or enrollment stage which is the process to collect the iris image and do preprocessing on iris image which the goal to remove unwanted data which include the segmentation step then pupil boundary detect step and Normalization the output of this step passing to feature extraction stage which is the process to extract the iris features, in this paper the wavelet decomposition methods are used to by extract the 1-D signal of the iris image by using wavelet decomposition , and the output of this stage is feature vector which store in database as template which used in matching propose . in another hand the testing stage which is the similar to training stage in Acquisition stage, preprocessing and feature extraction but the different is the output of feature extraction is passing to new stage which called matching stage which is the process to compare query image with template, finally the matching steps conducted to match training and testing ,the first iris code is divided into 2 subsets and the matching is done separately for each subset using Hamming Distance measure is used to find the distance between training and testing and passing the result to decision stage which decided the matching pair or non-matching pair. The result show the iris recognition by using wavelet decomposition can dramatically improve the accuracy of the system.

The evaluation of this system based on False Accept Rate (FAR) and False Reject Rate (FRR), the results are given in different dataset size of training and testing.

The future work of this paper may be to extend to study different feature extraction technique and matching technique which improve the performance of iris recognition system.

\section{REFERENCES}

[1] Anil K. jain, Arun A.Ross, Karthik Nanda kumar, 2011, "introduction to biometrics", Springer Sciences + Business Media, LLC, 233 Spring Street, New York ,NY 10013, USA

[2] Biometrics http://www.tml.tkk.fi/Opinnot/Tik110.501/1998/papers/12biometric/biometric.html

[3] Iris introduction available on this web: http://en.irisking.com/html/47/product 40

[4] J. G. Daugman, 1993 "high confidence visual recognition of persons by a test of statistical independence", IEEE transactions on pattern analysis and machine intelligence, $15(11), 11481160$

[5] L. Masek, 2003 , "Recognition of Human Iris Patterns for Biometric Identificationl"” , M.S. Dissertation, the University of Western Australia

[6] P. Verma, M. Dubey, P. Verma and S. Basu, 2012 , "Duaghman's algorithm method for iris recognition- a biometric approach",IJETAE international journal of emerging technology and advanced engineering, ISSN $2250-2459$, vol. 2 , issue 6

[7] E. Krichen, M.A. Mellakh, S. G. Salicetti and B. Dorizzi, 2004, "iris identification using wavelet packets" IEEE proceedings of the 17 th international conference on pattern recognition (ICPR04), vol. no 04

[8] Abikoye Oluwakemi C., Sadiku, J. S., Adewole Kayode S., and Jimoh Rasheed G, 2014,"'Iris Feature Extraction 
for Personal Identification using Fast Wavelet Transform (FWT)", International Journal of Applied Information Systems (IJAIS), ISSN : 2249-0868, Volume 6- No. 9.

[9] Yuqing He, Guangqin Feng, Yushi Hou, Li Li and Evangelia Micheli-Tzanakou, 2011 ,"Iris feature extraction method based on LBP and chunked encoding ", Seventh IEEE International Conference on Natural Computation 2011, 978-1-4244-9953-3

[10] J. Daugman, 2004, "How Iris Recognition Works," IEEE Trans.on Circuits and Systems for Video Technology, vol. 11, pp. 21-30.

[11] Wildes. R: 1997, "Iris recognition: an emerging biometric technology" . In: Proceedings of the IEEE, Vol. 85, No. 9.

[12] Boles W. W, Bolash. B: 1998, " A human identification technique using images of the iris and wavelet transform" . In: IEEE transactions on signal processing, Vol. 46, issue 4,1185-1188.
[13] Jain A, Bolle R and Pankanti S: 1999, “ Biometrics: Personal Identification in a Networked Society" . MA: Kluwer, Norwell,

[14] Daugman, J."How Iris Recognition Works",available at http://www.ncits.org/tc_home/m1htm/docs/m1020044.pd f.

[15] Daugman, J., 1993. "High Confidence Visual Recognition of Persons by a Test of Statistical Independence ,'IEEE transactions on pattern analysis and machine intelligence, vol. 15, no.11,pp. $1148-1161$

[16] M. M. H. Ali, V. H. Mahale, P. Yannawar and A. T. Gaikwad, 2016, "Fingerprint Recognition for Person Identification and Verification Based on Minutiae Matching," 2016 IEEE 6th International Conference on Advanced Computing (IACC), Bhimavaram, pp. 332-339 